\title{
COPACC: A Cooperative Proxy-Client Caching System for On-demand Media Streaming ${ }^{\star}$
}

\author{
Alan T.S. Ip ${ }^{1}$, Jiangchuan $\mathrm{Liu}^{2}$, and John C.S. Lui ${ }^{1}$ \\ 1 The Chinese University of Hong Kong, Shatin, N.T., Hong Kong \\ \{tsip, cslui\}@cse.cuhk.edu.hk \\ 2 Simon Fraser University, Vancouver, BC, Canada \\ csljc@ieee.org
}

\begin{abstract}
Proxy caching is a key technique to reduce transmission cost for on-demand multimedia streaming. However, its effectiveness is limited by the insufficient storage space and weak cooperations among proxies and their clients. In this paper, we propose COPACC, a novel cooperative proxy-and-client caching system that combines the advantages of both proxy caching and peer-to-peer (P2P) client communications. We propose a comprehensive suite of protocols to facilitate the interactions among different network entities in COPACC. We also develop an efficient cache allocation algorithm to minimize the aggregated transmission cost of the whole system. Simulation results demonstrate that COPACC achieves remarkably lower transmission cost. Moreover, it is much more robust than a pure $\mathrm{P} 2 \mathrm{P}$ system in the presence of node failures.
\end{abstract}

\section{Introduction}

Today's Internet has been increasingly used for carrying multimedia traffic, and on-demand streaming for clients is amongst the most popular networked media services. The limited server capacity, however, make efficient and scalable ondemand media streaming a challenging task. To reduce server/network loads, frequently used data is cached at proxies close to clients 1. Streaming media, particularly those with asynchronous demands, could benefit with a significant performance improvement from proxy caching given their static nature in content and highly localized access interests. Another approach is to generalize the proxy functionalities into every client 2. Such a P2P paradigm allows economical clients to contribute their storages for streaming. Video data originally provided by a server are spread among clients, thus amplifying the system capacity.

In this paper, we propose COPACC, a novel cooperative proxy-and-client caching system. We leverage the client-side caching to amplify the aggregated cache space and rely on dedicated proxies to effectively coordinate the communications. We develop an efficient cache allocation algorithm together with a comprehensive protocols suite to distribute video segments among the proxies

\footnotetext{
* This work is supported in part by the RGC Earmarked Grant.
} 
and clients such that the aggregated transmission cost is minimized. As most operations are executed by dedicated proxies, the system is resilient to client failures. We also embed an efficient indexing and searching algorithm for video contents cached across different proxies or clients. COPACC also makes effective use of multicast delivery, which further reduces the cost. The simulation results demonstrate that COPACC achieves remarkably lower transmission cost as compared to proxy-based caching with limited storage space. With the assistance from dedicated proxies, it is much more robust than a pure P2P system. Moreover, It scales well to larger networks, and the cost generally reduces when more proxies and clients cooperate with each other.

Fig. 1 depicts a generic architecture of COPACC. A cluster of proxies are logically connected to form overlay. The proxies and their clients are closely located with relatively low communication costs, while the proxies and the video server are located far away and incur higher costs. The video data are cached across proxies and clients of limited storage. As shown in Fig. 2, a video stream is partitioned into prefix and prefix-of-suffix. The proxies are responsible to cache the prefix of video, whereas the clients cache the prefix-of-suffix. Similar to 3 , this setting helps to minimize the initial playback latency. When a client expects to play a video, it initiates a playback request to its home proxy, which intercepts the request and computes a streaming schedule. It then fetches the prefix, prefixof-suffix, as well as the remaining part of suffix, and relays them to the client.

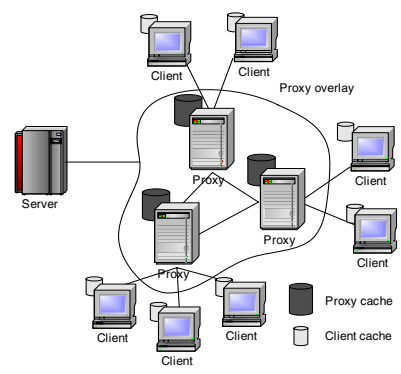

Fig. 1. The COPACC architecture

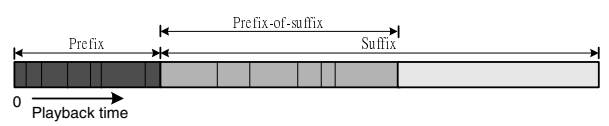

Fig. 2. Illustration of different portions of a video stream. The prefix is to be cached by proxies, while the prefix-of-suffix by clients

There are two key issues to be addressed: How to partition each video and allocate the prefixes and prefix-of-suffixes to different proxy and client? How to manage, search, and retrieve the cached data in different proxies and clients?

\section{Optimal Cache Allocation Problem (CAP)}

The optimal cache allocation problem (CAP) can be formulated as

CAP : $\min \operatorname{Cost}\left(\left\{p_{j}^{i}\right\},\left\{q_{j, k}^{i}\right\}\right)$,

s.t. $p_{j}^{i}, q_{j, k}^{i} \geq 0, j \in[1 \ldots H], k \in\left[1 \ldots K_{j}\right] ; \quad \sum_{i=1}^{N} p_{j}^{i} \leq s_{j}^{p} ; \quad \sum_{i=1}^{N} q_{j, k}^{i} \leq s_{j, k}^{c}$; $\sum_{j=1}^{H} p_{j}^{i}+\sum_{j=1}^{H} \sum_{k=1}^{K_{j}} q_{j, k}^{i} \leq V^{i}$, 
where $\operatorname{Cost}\left(\left\{p_{j}^{i}\right\},\left\{q_{j, k}^{i}\right\}\right)$ is the total transmission cost given prefix allocation $\left\{p_{j}^{i}\right\}$ and prefix-of-suffix allocation $\left\{q_{j, k}^{i}\right\}$; the second and third constraints follow the cache space limit of proxy $j$ and that of client $k$ of proxy $j$, respectively.

\section{1 $\quad$ Single Proxy with Client Caching}

We first consider a single proxy and multiple clients system. Since the transmission cost depends only on how the video are partitioned, we can assume that the caches of all the clients form an aggregated cache space, and derive the minimum transmission cost by finding the optimal values of $\left\{P^{i}\right\}$ and $\left\{Q^{i}\right\}$ subject to cache space constraints $S^{p}$ and $S^{c}$. We define an auxiliary cost function $C^{i}\left(P^{i}, Q^{i}\right)$, which is the cost for delivering video $i$ with prefix size $P^{i}$ and prefix-of-suffix size $Q^{i}$. Note that $\operatorname{Cost}\left(\left\{p_{j}^{i}\right\},\left\{q_{j, k}^{i}\right\}\right)$ is now equal to $\sum_{i=1}^{N} C^{i}\left(P^{i}, Q^{i}\right)$. The problem can then be solved by dynamic programming. It is applicable with arbitrary cost function $C^{i}\left(P^{i}, Q^{i}\right)$, which can be instantiated given a specific transmission scheme. As an example, assume both a server-to-client and a client-to-client transmissions are unicast-based and relayed by a proxy, $C^{i}\left(P^{i}, Q^{i}\right)$ can be derived as $\lambda f^{i} \cdot\left[w^{c \leftrightarrow p} P^{i}+2 w^{c \leftrightarrow p} Q^{i}+\left(w^{s \rightarrow p}+w^{c \leftrightarrow p}\right)\left(V^{i}-P^{i}-Q^{i}\right)+w^{i n}\left(P^{i}+Q^{i}\right)\right]$, where the first four terms in the square bracket represent the costs for retrieving prefix, prefix-of-suffix, the remaining suffix, and the internal cost of the proxy.

\subsection{Multiple Proxies with Client Caching}

We now consider the general COPACC system consisting multiple proxies and their respective clients. It involves interactions among several proxies and clients, and the unit transmission costs for the proxy-to-proxy and client-to-proxy links can be heterogeneous. In fact, we formally prove that CAP is NP-hard in this general case (see [4). We thus resort to a practically efficient heuristics, which consists of two phases: first, it partitions the prefix and prefix-of-suffix for each video; second, given the partitions, it allocates the segments of prefixes and prefix-of-suffixes to the proxies and clients.

1) Partitioning of prefix and prefix-of-suffix: In this phase, we approximate the system by a single proxy system with aggregated proxy cache space $S^{p}$ and aggregated client cache space $S^{c}$. An approximate solution of $\left\{P^{i}\right\}$ and $\left\{Q^{i}\right\}$ can be directly obtained using the dynamic programming algorithm.

2) Allocation to proxy and client caches: In this phase, we further partition the prefix and prefix-of-suffix, and allocate them to the proxies and clients. Since the allocation for prefixes to proxy caches is independent from that for prefixof-suffixes to client caches, we separate the two allocation problems and solve them individually. The optimal prefix allocation problem (PA) is formulated as

$$
\begin{aligned}
& \text { PA : } \min \sum_{i=1}^{N} \sum_{j=1}^{H} W^{p}\left(i, j, p_{j}^{i}\right) \\
& \text { s.t. } \sum_{j=1}^{H} p_{j}^{i}=P^{i}, i \in[1 \ldots N] ; \quad \sum_{i=1}^{N} p_{j}^{i} \leq s_{j}^{p}, j \in[1 \ldots H] .
\end{aligned}
$$

As $W^{p}\left(i, j, p_{j}^{i}\right)$ can be instantiated as $\sum_{j^{\prime}=1}^{H} p_{j}^{i}\left[w_{j, j^{\prime}}^{p \rightarrow p}+w_{j^{\prime}}^{c \leftrightarrow p}\right] \lambda_{j^{\prime}} f_{j^{\prime}}^{i}$ for unicast delivery, PA can be relaxed as a linear programming problem by re-writing it to $\min \sum_{i=1}^{N} \sum_{j=1}^{H} \bar{W}^{p}(i, j) \cdot p_{j}^{i}$. 
We should also consider the optimal suffix-of-prefix allocation problem (SA) for client cache. Obviously, both the problem SA and the cost function itself have similar structure as that of problem PA. Thus, we omit the derivation of SA here. Note that the linear programming relaxation also applies for SA. The optimizations shown above can also be applied to multicast delivery (see [4).

\section{The Cooperative Proxy-Client Caching Protocol}

As shown in Fig. 1, COPACC operates as a two-level overlay, where the first level consists of all the proxies, and the second level consists of each proxy and its own clients. The interactions among different entities in this two-level overlay are specified by a cooperative proxy-client caching protocol, which consists of three subprotocols. 1) Cache allocation and organization protocol specifies the election of proxy coordinator, which executes the optimal cache allocation algorithm and disseminates the lookup information using simplest hashing. 2) Cache lookup and retrieval protocol defines the discovery and retrieval of cache between proxies. 3) Client access and integrity verification protocol performs verification operation, which detects forged video data through a simple yet effective signature-based verification algorithm. The details of this protocol suite can be found in [4].

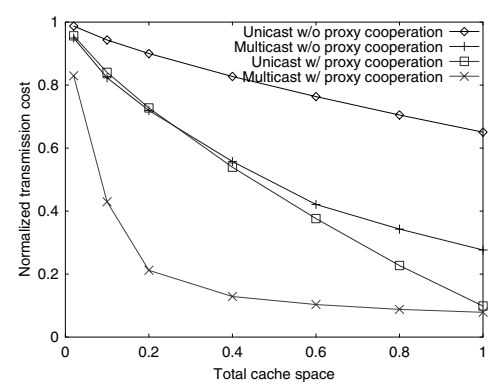

Fig. 3. Transmission cost as a function of the total proxy-client cache space

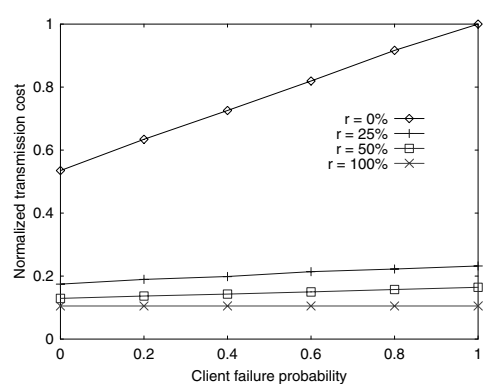

Fig. 4. Transmission cost versus client failure probability

\section{Performance Evaluation}

A primary design objective of COPACC is to reduce the transmission cost. Fig. 3 plots the transmission cost as a function of the total cache space, where the proxies and clients respectively contribute half of the total cache size. The cache sizes are normalized by the total size of the video repository, and the transmission costs are normalized by the corresponding cost of a system with no cache. Not surprisingly, increasing the total space reduces transmission cost. With unicast, the cost decreases linearly, while with suffix multicast, it decreases much faster. When the total cache space is 0.2 , the cost with suffix multicast has been 
reduced to 0.2 ; in other words, a $20 \%$ cache space leads to a $80 \%$ cost reduction, which implies that batching the requests from local clients can avoid a significant amount of remote transmissions. It is also clear that the cost with cooperative proxies is much lower, particularly when multicast is also enabled in local paths.

The robustness in the presence of client failures is also a critical concern in COPACC. In Fig. 4, we show the transmission cost as a function of different client failure probabilities. We vary, $r$, the fraction of the total proxy cache space in the total cache space from $0 \%$ to $100 \%$. When $r=0 \%$, COPACC degenerates to a pure P2P system, and, when $r=100 \%$, it degenerates to a pure proxy-based system. We can see that, when there is no client failure, the costs for different $r$ are quite close if there are certain cache existed in proxies. More importantly, the cost of the pure proxy-based system remains unchanged when increasing client failures, and that for $0 \%<r<100 \%$ is also very stable. For illustration, even if $r$ is $25 \%$, the transmission cost only slightly increases with an increase of failure probability; when the failure probability is 1 , the cost remains a low as 0.22 . To the contrary, the cost of the pure P2P system quickly increases and reaches 1 (the cost of a zero-cache system), when all clients fail. Such results demonstrate that the use of dedicated proxies with suffix batching remarkably improves the robustness and resilience of COPACC in the presence of client failures.

In summary, COPACC effectively utilizes the client-side cache space and realizes a low-cost video streaming system. Yet with the assistance of dedicated proxies, it is quite robust to accomodate dyanmic and even malicious clients. More results supporting such arguments as well as the behavior of COPACC under diverse network configurations can be found in [4].

\section{References}

1. Liu, J., Xu, J.: Proxy Caching for Media Streaming over the Internet. IEEE Communications (2004)

2. Cui, Y., Li, B., Nahrstedt, K.: oStream: Asynchronous Streaming Multicast in Application-Layer Overlay Networks. IEEE JSAC 22 (2004)

3. Wang, B., Sen, S., Adler, M., Towsley, D.: Optimal Proxy Cache Allocation for Efficient Streaming Media Distribution. In: Proc. IEEE INFOCOM'02, NY (2002)

4. Ip, A.T.S., Liu, J., Lui, J.C.S.: COPACC: A Cooperative Proxy-Client Caching System for On-Demand Media Streaming. Technical Report (2004, CUHK, http://www.cs.sfu.ca/ jcliu/Papers/TR-COPACC-Final/TR-COPACC.pdf) 